\title{
Probing the matter power spectrum with the galaxy clustering ratio
}

Julien Bel*

Osservatorio Astronomico di Brera

E-mail: julien.bel.20@gmail.com

The galaxy clustering ratio [1,2] is a new clustering statistic that provides access to characteristic parameters of the power spectrum of mass density fluctuations without the need to specify the galaxy biasing function nor a model for peculiar velocity distortions. I will demonstrate the method using galaxy simulations as well as real redshift data (from the VIPERS and the SDSS surveys) and show that it allows to fix the reduced density of matter to a precision of nearly $6 \%$. I will argue that this approach will be instrumental in searching for evidences of new physics beyond the standard model of cosmology and in planning future redshift surveys such as EUCLID. References

[1] J. Bel, C. Marinoni, 2014, A\&A, 563, 36

[2] J. Bel, C. Marinoni, B. Granett et al. (the VIPERS collaboration), 2014, A\&A 563, 37

Frontiers of Fundamental Physics 14 - FFP14,

15-18 July 2014

Aix Marseille University (AMU) Saint-Charles Campus, Marseille

* Speaker. 